\title{
Influence of light nuclei on neutrino-driven supernova outflows
}

\author{
A. Arcones, ${ }^{1,2}$ G. Martínez-Pinedo, ${ }^{2}$ E. O'Connor, ${ }^{3,4}$ A. Schwenk, ${ }^{3}$ H.-Th. Janka, ${ }^{5}$ C. J. Horowitz, ${ }^{6}$ and K. Langanke ${ }^{1,2}$ \\ ${ }^{1}$ Institut für Kernphysik, TU Darmstadt, Schlossgartenstr. 9, D-64289 Darmstadt, Germany \\ ${ }^{2}$ Gesellschaft für Schwerionenforschung, Planckstr. 1, D-64291 Darmstadt, Germany \\ ${ }^{3}$ TRIUMF, 4004 Wesbrook Mall, Vancouver, British Columbia, V6T 2A3, Canada \\ ${ }^{4}$ Department of Physics, California Institute of Technology, Pasadena, California 91125, USA \\ ${ }^{5}$ Max-Planck-Institut für Astrophysik, Karl-Schwarzschild-Str. 1, D-85741 Garching, Germany \\ ${ }^{6}$ Nuclear Theory Center and Department of Physics, Indiana University, Bloomington, Indiana 47408, USA
}

(Received 24 May 2008; published 24 July 2008)

\begin{abstract}
We study the composition of the outer layers of a protoneutron star and show that light nuclei are present in substantial amounts. The composition is dominated by nucleons, deuterons, tritons and alpha particles; ${ }^{3} \mathrm{He}$ is present in smaller amounts. This composition can be studied in laboratory experiments with new neutron-rich radioactive beams that can reproduce similar densities and temperatures. After including the corresponding neutrino interactions, we demonstrate that light nuclei have a small impact on the average energy of the emitted electron neutrinos, but are significant for the average energy of antineutrinos. During the early post-explosion phase, the average energy of electron antineutrinos is slightly increased, while at later times during the protoneutron star cooling it is reduced by about $1 \mathrm{MeV}$. The consequences of these changes for nucleosynthesis in neutrino-driven supernova outflows are discussed.
\end{abstract}

DOI: 10.1103/PhysRevC.78.015806

PACS number(s): 26.50.+x, 26.30.-k, 25.30.Pt, 97.60.Bw

\section{INTRODUCTION}

Core-collapse supernovae are the birth places of neutron stars. During the explosion, around $10^{53}$ ergs, corresponding to the binding energy of the neutron star, are emitted in all neutrino species from a thermal surface denoted as neutrinosphere. The spectrum and luminosities of the neutrinos radiated from the newly formed neutron star, the so-called protoneutron star, are of great importance for the interpretation of supernova neutrino detections, for studies of flavor conversion in the star mantle [1,2], and for nucleosynthesis occurring in the so-called neutrino-driven wind $[3,4]$.

A crucial input for protoneutron star evolution is the equation of state (EOS). Currently available simulations use either the Lattimer and Swesty [5] or Shen et al. [6] EOS, or an EOS based on nuclear statistical equilibrium (NSE) [7]. All these EOS describe the nucleonic composition by a mixture of neutrons, protons, alpha particles, and a representative heavy nucleus. While these EOS include the essential composition for the relatively low densities and high temperatures present in the protoneutron star atmosphere, where matter is fully dissociated, they cannot account for the composition in the region of the crust where the larger densities $\left(\rho \sim 10^{12} \mathrm{~g} / \mathrm{cm}^{3}\right)$ allow for the formation of light nuclei with $A=2$ and 3 in addition to alpha particles [8,9]. In contrast to the conditions in cold neutron stars, the high temperatures $(T \gtrsim 4 \mathrm{MeV})$ of the protoneutron star crust suppress the formation of heavier nuclei. Deeper in the protoneutron star the densities become so large that light nuclei melt and a transition from inhomogeneous phases to homogeneous nuclear matter takes place $[10,11]$. As we will discuss below, the change in composition caused by the presence of light nuclei in the outer crust affects the neutrino opacities and consequently changes their spectra and luminosities. In addition, light nuclei are present in the region behind the shock, where the emitted neutrinos are expected to deposit their energy in the delayed supernova explosion mechanism [12,13], and therefore interactions with neutrinos have to be included as well $[8,9,14]$.

The neutrinos emitted by the young protoneutron star produce an outflow of baryonic matter known as the neutrinodriven wind that has been the subject of many studies including full hydrodynamical simulations [7], analytical [4] and steady-state approaches (see Ref. [15] and references therein). This outflow is initially very hot and essentially consists of free neutrons and protons in a ratio that is determined by the competition of neutrino and antineutrino absorptions on nucleons and their inverse reactions. But as the matter expands and cools, nucleons can be assembled into nuclei, and elements even heavier than iron can be formed. If this occurs with a large abundance of free neutrons present, these can be captured on heavy nuclei (the "seed") producing an r-process [16,17]. For a successful r-process a large neutron-to-seed ratio is necessary, requiring outflows with short dynamical time scales (a few milliseconds), high entropies (above $150 k_{\mathrm{B}}$ ) and low electron fractions $\left(Y_{e}<0.5\right)$ [18].

In the present paper, we explore the influence of light nuclei on the spectra and luminosities of electron neutrinos and antineutrinos emitted during the cooling phase of the protoneutron star and consequently on the electron-to-baryon ratio of the ejected matter. The existence of light nuclei potentially also affects $\mu$ and $\tau$ neutrinos. We plan to investigate this in future work. The early post-bounce evolution and pre-explosion phase might also be affected by the presence of light nuclei in the matter composition. However, this issue cannot be explored here in detail, because the neutrino transport conditions in those phases and the development of hydrodynamical instabilities in the forming neutron star and in the neutrino heating region (for reviews, see Refs. [13,19]) 
require full radiation hydrodynamics simulations, which are beyond our study here.

If light nuclei are present in the region near the neutrinospheres they will influence neutrinos and antineutrinos differently. Deep in the interior of the protoneutron star neutrinos, are in chemical equilibrium with matter. At larger radii the decrease of temperature and density allows for neutrinos to decouple from matter near the protoneutron star surface and near the region where nuclei form. For the very neutron-rich conditions present in this environment, the formation of light nuclei occurs mostly at the expense of free protons. As protons represent the major source of opacity for electron antineutrinos, and because the antineutrino cross sections on protons and light nuclei are different for the relevant energies, light nuclei can potentially affect the spectra and luminosities of the emitted antineutrinos. The situation is not the same for electron neutrinos. The abundance of neutrons is so large that it is insignificantly changed by the appearance of light nuclei. Consequently, the electron neutrino opacity, which is dominated by interactions with neutrons, remains practically unchanged. This asymmetrical effect on the radiated neutrinos and antineutrinos can potentially change the proton-to-neutron ratio of the ejecta and consequently the nucleosynthesis. A study of these effects requires an EOS that includes light nuclei in the composition and their corresponding neutrino cross sections.

This warm nuclear system near the neutrinosphere, including the abundances of light nuclei, can be studied with heavy-ion collisions. Fragments emitted from a system at intermediate velocities may come from a low-density region between the colliding nuclei. The density and temperature of this region can be similar to conditions near the neutrinosphere. Kowalski et al. [20] have measured the abundances of light nuclei (deuterons, tritons, ${ }^{3} \mathrm{He}$, and ${ }^{4} \mathrm{He}$ ) in near Fermi energy heavy-ion collisions of ${ }^{64} \mathrm{Zn}$ on ${ }^{92} \mathrm{Mo}$ and on ${ }^{197} \mathrm{Au}$. They found ${ }^{4} \mathrm{He}$ abundances and symmetry energies similar to those predicted by the virial EOS [21]. In the future, experiments with radioactive beams will enable studies of the more neutronrich neutrinosphere conditions.

This paper is organized as follows. Section II presents the EOS (which we use for the determination of light-element abundances), the neutron star atmosphere model, and the neutrino cross sections with light nuclei. Section III discusses the impact of light nuclei on the spectra and luminosities of electron neutrinos and antineutrinos and their influence on the electron fraction of the ejecta. Finally, we conclude in Sec. IV.

\section{EQUATION OF STATE, OPACITIES, AND NEUTRINOSPHERE DETERMINATION}

\section{A. Neutron star model and equation of state}

Our work employs the protoneutron star model M15-11-r1 of Ref. [7]. The model describes the structure of the surface layers and of the neutrino-driven wind of a protoneutron star with a baryonic mass of $1.4 \mathrm{M}_{\odot}$, obtained in a spherically symmetric simulation of the (parametrized) neutrino-driven explosion of a $15 \mathrm{M}_{\odot}$ star. The thermodynamical state of hot, dense matter including its baryonic composition is fully

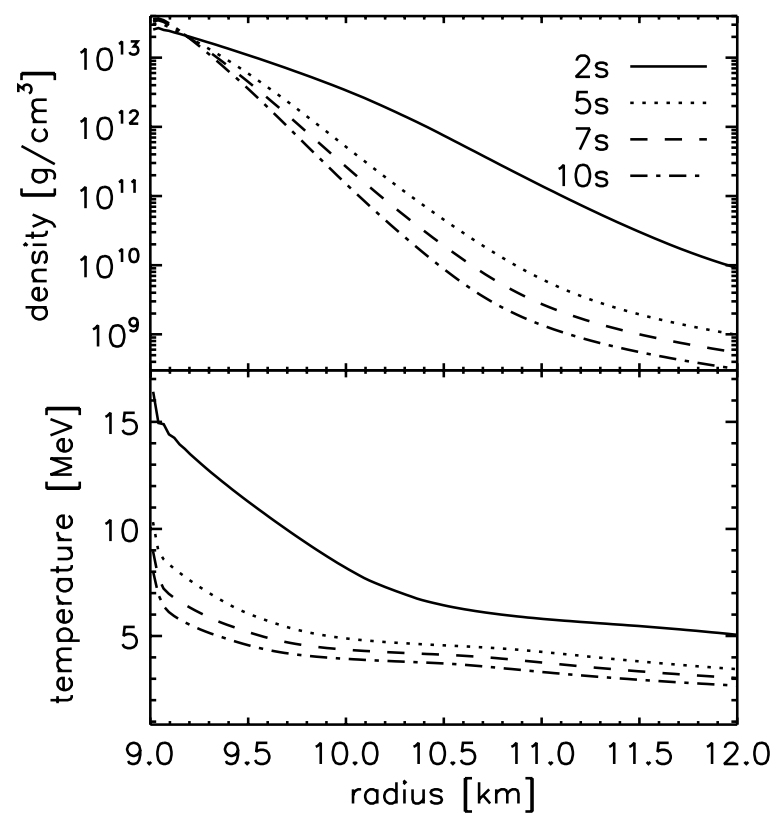

FIG. 1. Neutron star atmosphere profiles of density and temperature corresponding to model M15-11-r1 of Ref. [7] for times $t=2,5,7$ and $10 \mathrm{~s}$ post bounce.

characterized by three independent variables, for example, density, temperature, and electron chemical potential (or baryon density, energy density, and electron fraction). In the supernova context, these have to be determined by solving the equations of hydrodynamics for the stellar plasma and the transport equations for neutrinos and antineutrinos of all flavors, making use of an EOS that relates the pressure and all other thermodynamic variables to the three basic ones. Figure 1 shows the time evolution of the density and temperature profiles after core bounce in the region around the neutrinospheres ("neutron star atmosphere") as predicted by model M15-11-r1. We point out that this model did not include light elements, therefore the temperature and density are not exactly those one would obtain if light elements were included.

Using these temperature and density profiles, we can determine the electron chemical potential (or, equivalently, the electron fraction $Y_{e}$ ) by making the assumption that the matter is in (neutrinoless) beta equilibrium. This is fairly well fulfilled in the neutron star atmosphere at sufficiently late postbounce times when the deleptonization of the protoneutron star interior has slowed down and the nascent neutron star radiates electron neutrinos and antineutrinos with very similar number luminosities. In this case the chemical potentials of neutrons, protons, and electrons fulfill the equality $\mu_{n}=\mu_{p}+\mu_{e}$, which allows one to compute $\mu_{e}$ by invoking also charge neutrality, $Y_{e}=Y_{p}$, and the relation for the total baryon number, $Y_{p}=1-Y_{n}$. Here $Y_{p}$ and $Y_{n}$ denote the number fractions of free plus bound protons and neutrons, respectively. It is clear that the $Y_{e}$-profile one thus obtains will depend on the considered EOS.

We will compare results obtained with three different EOS. The first is the one used in Ref. [7], which essentially describes the baryonic composition as a noninteracting Boltzmann gas of neutrons, protons, and alpha particles in NSE (a representative 
heavy nucleus is also included but of no relevance here). Calculations performed with this EOS serve as the reference to which we compare our results. The second EOS, denoted by NSE EOS, also assumes matter in NSE but consists of several thousand nuclei, for which partition functions have been computed in Ref. [24], and includes Coulomb corrections $[25,26]$ and Fermi-Dirac statistics for neutrons and protons. Our third EOS is the virial EOS [8,21-23], which is based on nuclei with $A \leqslant 4$ and their interactions through second virial coefficients derived directly from scattering phase shifts. Figure 2 shows the composition obtained from these different EOS for the thermodynamical conditions of Fig. 1, assuming beta equilibrium for each of the displayed cases. In agreement with our arguments, we observe that in the vicinity of the neutrinospheres the virial and NSE EOS predict deuteron and triton mass fractions significantly larger than the one of free protons.

The virial and NSE EOS lead to very similar compositions up to densities $\rho \sim 10^{13} \mathrm{~g} / \mathrm{cm}^{3}$. At these densities, the treatment of nuclear interactions in the virial EOS becomes unreliable, and in the NSE EOS interactions are neglected. This is signaled by a sudden increase in the abundance of heavy nuclei, when using the NSE EOS, or by a sudden drop of the proton mass fraction in the virial EOS, due to the breakdown of the virial expansion with only second virial coefficients. For densities lower than $\rho \sim 10^{13} \mathrm{~g} / \mathrm{cm}^{3}$, we observe that the main differences of the virial and NSE EOS are in the alpha particle mass fractions due to attractive nucleon-alpha interactions [21].

\section{B. Neutrino opacities and neutrinospheres}

To determine the neutrinosphere radius we follow Refs. [27,28] and define the effective neutrino opacity for energy exchange or thermalization by

$$
\kappa_{\mathrm{eff}}=\sqrt{\kappa_{\mathrm{abs}}\left(\kappa_{\mathrm{abs}}+\kappa_{\mathrm{scatt}}\right)} .
$$

The absorption opacity $\kappa_{\text {abs }}$ is considered to include all processes in which neutrinos exchange energy with the stellar medium, while the scattering opacity $\kappa_{\text {scatt }}$ contains those processes where mostly the momentum of the neutrinos is changed but essentially not their energy. These opacities, $\kappa=\sum n_{i} \sigma_{i}$, are obtained from the neutrino cross sections $\sigma_{i}$ and number densities $n_{i}$ of the target particles in the stellar plasma.

In order to determine an average neutrinosphere radius, rather than an energy-dependent one, we assume neutrinos to be in thermal equilibrium with matter up to their so-called average energy sphere, where the bulk of the neutrino spectrum begins to decouple thermally from the background medium of the star. We will consider this energy sphere as the appropriate neutrinosphere in the context of the work presented in this paper. Until this location the neutrino phase-space distribution will be assumed to be a Fermi-Dirac distribution function for the local gas temperature. Outside of the neutrinosphere the spectral temperature is taken to be fixed to its value at the neutrinosphere. Thus, we average all opacities over the relevant neutrino spectral distributions and define

$$
\left\langle\kappa_{\text {eff }}\right\rangle=\sqrt{\left\langle\kappa_{\text {abs }}\right\rangle\left(\left\langle\kappa_{\text {abs }}\right\rangle+\left\langle\kappa_{\text {scatt }}\right\rangle\right)} .
$$

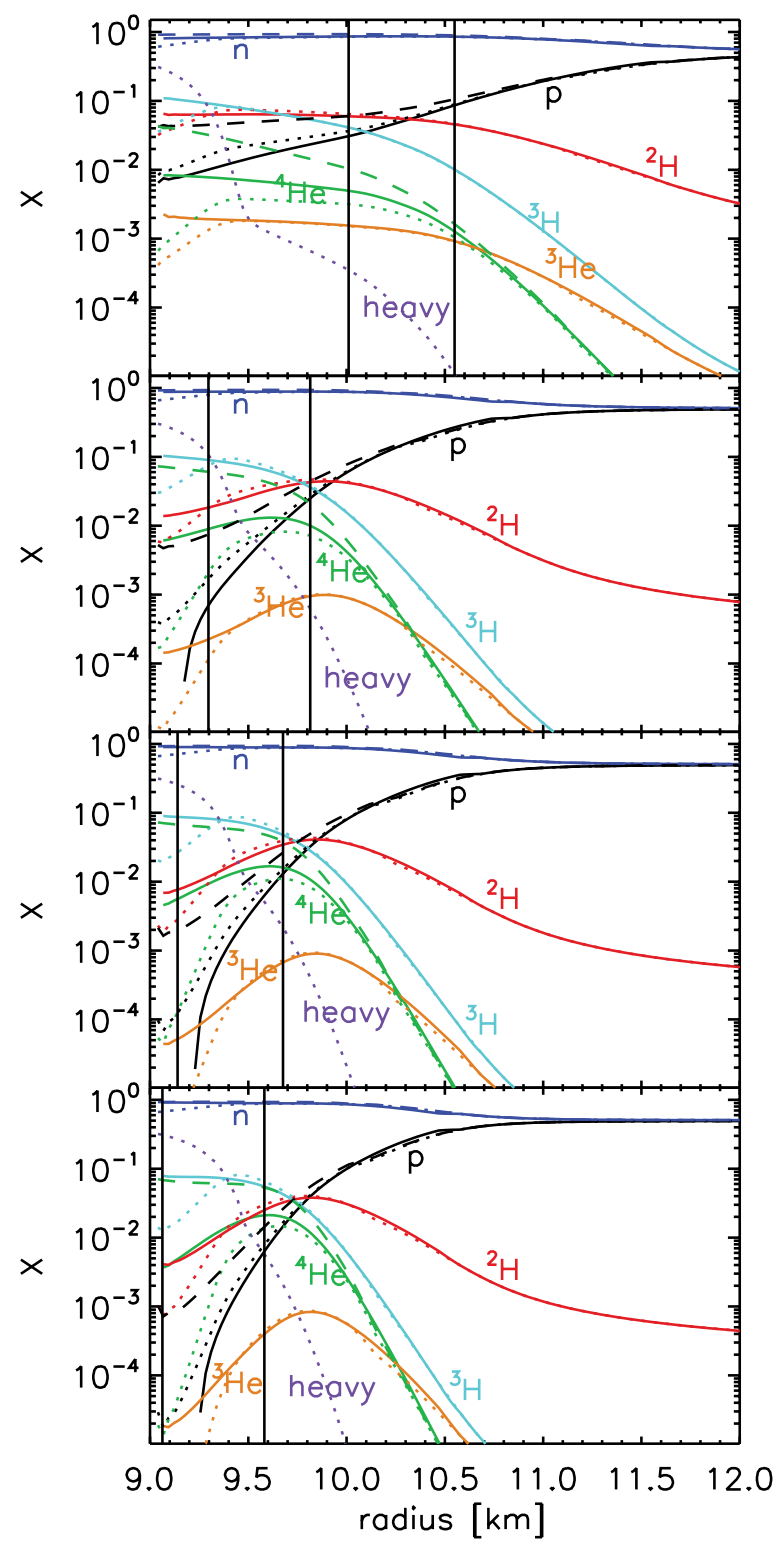

FIG. 2. (Color online) Mass fractions (defined as the mass density of species $i$ divided by the total mass density) for nuclei present around the surface of the protoneutron star using three different EOS: the one used in Ref. [7] (dashed lines), the NSE EOS (dotted lines), and the virial EOS [8,21-23] (solid lines). Beta equilibrium was assumed in each case. The lines labeled "heavy" represent the mass fraction of nuclei with $A>4$, which are included in the NSE EOS but not in the others. From top to bottom the profiles correspond to $t=2,5,7$ and $10 \mathrm{~s}$ post bounce. The vertical lines mark the positions of the neutrinospheres of electron neutrinos (right line) and electron antineutrinos (left line).

In the calculation of $\kappa_{\text {scatt }}$ we include elastic scattering off nucleons [29] and nuclei [30] for both electron neutrinos and antineutrinos. For determining $\kappa_{\mathrm{abs}}$ for electron neutrinos, it is sufficient to consider their absorption on neutrons, because neutrons dominate the composition by far. In the case of electron antineutrinos, besides weak processes [29] with the rare protons we have to include also inverse nucleon-nucleon bremsstrahlung [31] and in particular charged-current and 
neutral-current interactions with deuterons [32] and tritons (as discussed below). The situation is different for ${ }^{3} \mathrm{He}$ as target particle. Its abundance is always very low so that this contribution to the antineutrino opacity can be neglected. In addition, we also consider neutrino and antineutrino inelastic scattering on electrons and positrons. However, its contribution to the total opacity is very small (Fig. 3) as the cross section for these processes [33] is smaller than those for baryonic targets, due to the linear instead of quadratic energy dependence. Therefore we neglect their contributions. Moreover, we neglect neutrino-antineutrino annihilation as both the cross section and target density are smaller than those for interactions with baryonic targets. Ignoring these processes has hardly any influence on the relative changes of the neutrinospheric positions that we intend to discuss in this paper. Our approach for estimating the influence of composition effects in the surface layers of nascent neutron stars on the position of neutrinomatter decoupling is rather qualitative and approximative anyway. It is certainly not suitable for making exact quantitative predictions, a goal that definitely requires radiationhydrodynamics simulations with energy-dependent neutrino transport.

The first $a b$ initio calculations for neutral-current inelastic cross section off ${ }^{3} \mathrm{H}$ and ${ }^{3} \mathrm{He}$ nuclei were presented in Ref. [8], where the neutrino energy was averaged over a Fermi-Dirac spectrum for given temperature. For our purpose, it is advantageous to have the total cross sections as a function of neutrino energy. In addition, charged-current cross sections for antineutrinos on tritons are needed. Therefore, we have computed the relevant cross sections using a model based on the random phase approximation (RPA) which has been successfully applied to the study of many neutrino-induced reactions (for example, see Refs. [34,35]). Our approach follows the one described in Ref. [36], where we adopt an RPA that distinguishes between proton and neutron degrees of freedom for the particle and hole states. The parent ground state is approximated by the lowest independent particle model state with the single-particle energies derived from an appropriate Woods-Saxon potential that reproduces the particle separation energies in the parent nucleus. The partial occupancy formalism as described in Ref. [36] is applied to the proton and neutron states and holes for ${ }^{3} \mathrm{H}$ and ${ }^{3} \mathrm{He}$, respectively. As residual interaction, we have used the Landau-Migdal force of Ref. [36]. The calculation includes all multipole transitions with $\lambda \leqslant 4$ and both parities, properly accounting for the dependence of the multipole operators on the momentum transfer [37,38]. The Gamow-Teller strength has been quenched by a factor 0.74 as in shell-model calculations [39]. Our calculated cross sections are listed in Table I as a function of incident antineutrino energy $E$. To compare our results with the ab initio calculations of Ref. [8], we have folded the cross sections with a Fermi-Dirac neutrino distribution and find agreement to better than a few percent in all cases.

From the effective opacity of Eq. (2), we determine the optical depth as

$$
\tau(r)=\int_{r}^{\infty}\left\langle\kappa_{\mathrm{eff}}\right\rangle d r .
$$

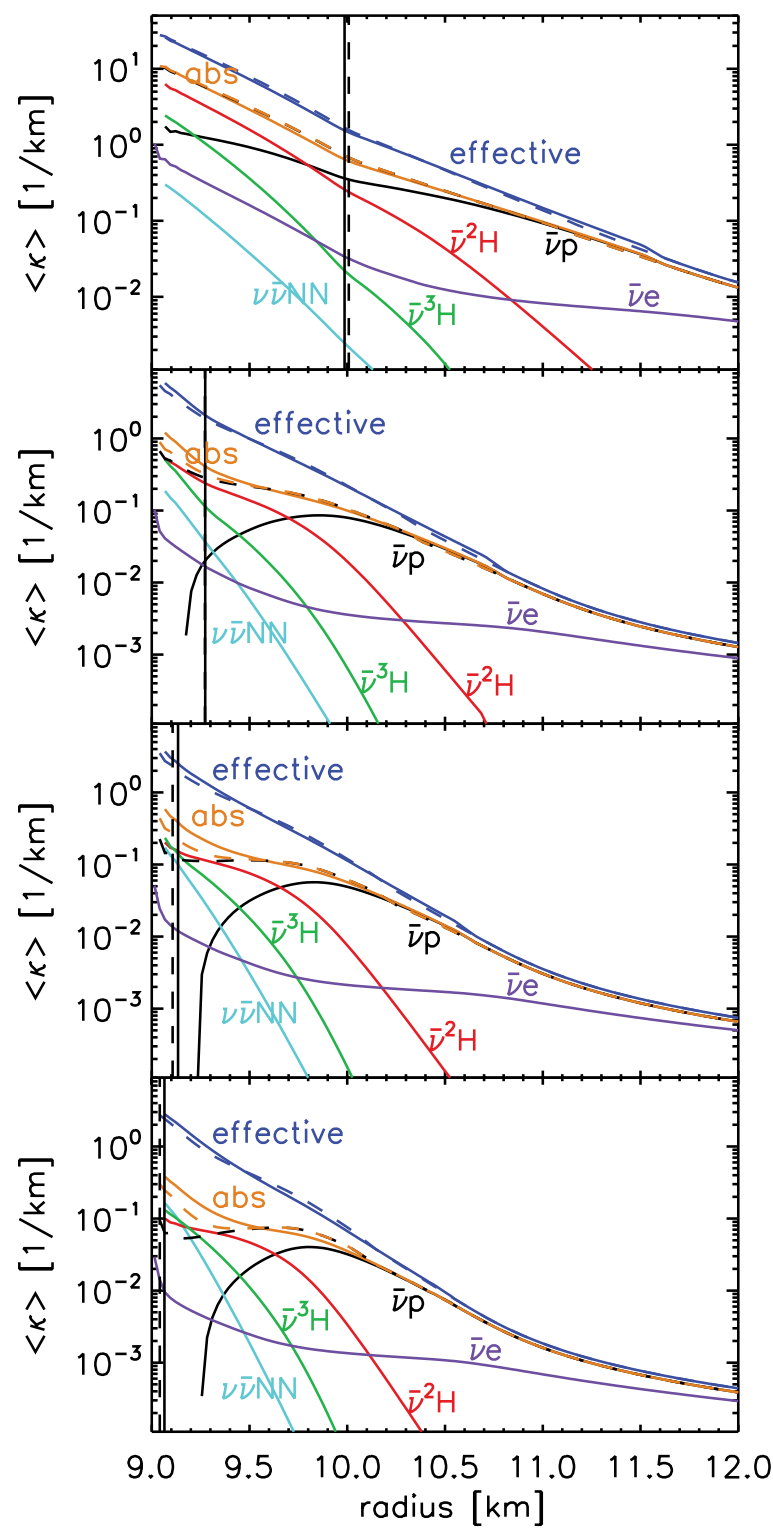

FIG. 3. (Color online) Different contributions to the absorption opacity $\left\langle\kappa_{\text {abs }}\right\rangle$ and effective opacity $\left\langle\kappa_{\text {eff }}\right\rangle$ of electron antineutrinos as a function of radius for $t=2,5,7$ and $10 \mathrm{~s}$ post bounce (from top to bottom) and for two different EOS: the one used in Ref. [7] (dashed lines) and the virial EOS (solid lines), based on the target abundances displayed in Fig. 2. In addition, the total absorption ("abs") and total effective opacity ("effective") are shown. The vertical lines mark the positions of the neutrinospheres of electron antineutrinos for the two different EOS. In the second panel from the top the vertical dashed and solid lines coincide.

The neutrinospheric radius $R_{v}$ is then defined as the position where the optical depth reaches $\tau\left(R_{\nu}\right)=2 / 3$ [40]. We assume that the neutrino distribution function at the neutrinosphere is represented by a Fermi-Dirac spectrum of temperature $T$ and effective degeneracy parameter $\eta_{\nu}$ :

$$
\frac{d n_{v}}{d E}(E)=\frac{1}{2 \pi^{2}(\hbar c)^{3}} \frac{E^{2}}{\exp \left[E /\left(k T_{\nu}\right)-\eta_{v}\right]+1} .
$$


TABLE I. Cross sections for charged-current and neutral-current antineutrino scattering off ${ }^{3} \mathrm{H}$ as a function of incident antineutrino energy $E$.

\begin{tabular}{|c|c|c|}
\hline$E[\mathrm{MeV}]$ & ${ }^{3} \mathrm{H}\left(\bar{v}_{e}, e^{+}\right)\left[10^{-42} \mathrm{~cm}^{2}\right]$ & ${ }^{3} \mathrm{H}\left(\bar{v}_{e}, \bar{v}_{e}^{\prime}\right)\left[10^{-42} \mathrm{~cm}^{2}\right]$ \\
\hline 11 & $1.66 \times 10^{-5}$ & $2.64 \times 10^{-5}$ \\
\hline 12 & $9.56 \times 10^{-5}$ & $4.30 \times 10^{-4}$ \\
\hline 13 & $2.40 \times 10^{-4}$ & $1.69 \times 10^{-3}$ \\
\hline 14 & $4.49 \times 10^{-4}$ & $4.32 \times 10^{-3}$ \\
\hline 15 & $1.79 \times 10^{-3}$ & $8.90 \times 10^{-3}$ \\
\hline 16 & $4.97 \times 10^{-3}$ & $1.60 \times 10^{-2}$ \\
\hline 17 & $1.13 \times 10^{-2}$ & $2.64 \times 10^{-2}$ \\
\hline 18 & $2.19 \times 10^{-2}$ & $4.08 \times 10^{-2}$ \\
\hline 19 & $3.80 \times 10^{-2}$ & $6.01 \times 10^{-2}$ \\
\hline 20 & $6.13 \times 10^{-2}$ & $8.52 \times 10^{-2}$ \\
\hline 21 & $9.32 \times 10^{-2}$ & $1.17 \times 10^{-1}$ \\
\hline 22 & $1.35 \times 10^{-1}$ & $1.57 \times 10^{-1}$ \\
\hline 23 & $1.90 \times 10^{-1}$ & $2.05 \times 10^{-1}$ \\
\hline 24 & $2.58 \times 10^{-1}$ & $2.64 \times 10^{-1}$ \\
\hline 25 & $3.43 \times 10^{-1}$ & $3.34 \times 10^{-1}$ \\
\hline 26 & $4.46 \times 10^{-1}$ & $4.17 \times 10^{-1}$ \\
\hline 27 & $5.70 \times 10^{-1}$ & $5.14 \times 10^{-1}$ \\
\hline 28 & $7.18 \times 10^{-1}$ & $6.26 \times 10^{-1}$ \\
\hline 29 & $8.93 \times 10^{-1}$ & $7.54 \times 10^{-1}$ \\
\hline 30 & $1.10 \times 10^{0}$ & $9.01 \times 10^{-1}$ \\
\hline 32 & $1.61 \times 10^{0}$ & $1.25 \times 10^{0}$ \\
\hline 34 & $2.28 \times 10^{0}$ & $1.70 \times 10^{0}$ \\
\hline 36 & $3.13 \times 10^{0}$ & $2.24 \times 10^{0}$ \\
\hline 38 & $4.20 \times 10^{0}$ & $2.89 \times 10^{0}$ \\
\hline 40 & $5.50 \times 10^{0}$ & $3.66 \times 10^{0}$ \\
\hline 42 & $7.08 \times 10^{0}$ & $4.56 \times 10^{0}$ \\
\hline 44 & $8.97 \times 10^{0}$ & $5.60 \times 10^{0}$ \\
\hline 46 & $1.12 \times 10^{1}$ & $6.78 \times 10^{0}$ \\
\hline 48 & $1.38 \times 10^{1}$ & $8.13 \times 10^{0}$ \\
\hline 50 & $1.69 \times 10^{1}$ & $9.63 \times 10^{0}$ \\
\hline 54 & $2.43 \times 10^{1}$ & $1.31 \times 10^{1}$ \\
\hline 58 & $3.38 \times 10^{1}$ & $1.74 \times 10^{1}$ \\
\hline 62 & $4.56 \times 10^{1}$ & $2.23 \times 10^{1}$ \\
\hline 66 & $5.99 \times 10^{1}$ & $2.81 \times 10^{1}$ \\
\hline 70 & $7.69 \times 10^{1}$ & $3.45 \times 10^{1}$ \\
\hline 74 & $9.65 \times 10^{1}$ & $4.17 \times 10^{1}$ \\
\hline 78 & $1.18 \times 10^{2}$ & $4.96 \times 10^{1}$ \\
\hline 82 & $1.42 \times 10^{2}$ & $5.82 \times 10^{1}$ \\
\hline 86 & $1.69 \times 10^{2}$ & $6.75 \times 10^{1}$ \\
\hline 90 & $1.98 \times 10^{2}$ & $7.74 \times 10^{1}$ \\
\hline 95 & $2.37 \times 10^{2}$ & $9.05 \times 10^{1}$ \\
\hline 100 & $2.80 \times 10^{2}$ & $1.04 \times 10^{2}$ \\
\hline
\end{tabular}

The neutrino number luminosity [41] at the neutrinosphere is then given by

$$
L_{n, v}=\frac{2 c}{\pi(\hbar c)^{3}}\left(k T_{v}\right)^{3} R_{v}^{2} f\left(R_{v}\right) F_{2}\left(\eta_{v}\right)
$$

and a similar equation for antineutrinos. Here $f$ is the flux factor needed to convert number density to number flux, with $f\left(R_{v}\right) \approx 0.25$ at the location of the neutrinosphere [41]. The Fermi integral for relativistic particles is defined as $F_{n}(\eta)=$ $\int_{0}^{\infty} d x x^{n} /[1+\exp (x-\eta)]$, where $\eta$ should be regarded as a spectral parameter that is not necessarily related to the neutrino degeneracy.

The above procedure allows for a completely independent determination of the electron neutrino and antineutrino spheres, and consequently of their number luminosities. However, the number luminosities are constrained by the requirement that the net flux of electron neutrinos minus electron antineutrinos is positive or zero to warrant that the lepton content of the star does not increase. Simulations [42] show that the nascent neutron star deleptonizes only very gradually and therefore the number luminosities of neutrinos and antineutrinos are rather similar, allowing us to make the approximation that the net flux is equal to zero, $L_{n, v}=$ $L_{n, \bar{v}}$. This choice is consistent with our assumption of beta equilibrium for fixing the composition in the outer layers of the neutron star (see Sec. II A), in which case the same numbers of electron neutrinos and antineutrinos are created in this region. Since our calculations are mainly sensitive to the difference in the spectral properties of neutrinos and antineutrinos, we set $\eta_{\bar{v}}=0$ for simplicity and obtain $\eta_{v}$ from the condition of zero net flux.

We use the following iterative procedure [43] to determine the $v_{e}$ and $\bar{v}_{e}$ neutrinospheres. First, we assume some initial values for the temperatures of the neutrino and antineutrino distributions, and determine $\eta_{v}$ from the condition of zero net flux. Using these values we compute the radii, at which the optical depths become $2 / 3$. Then, we set the new $v_{e}$ and $\bar{v}_{e}$ temperatures to the local temperature at the corresponding radii and update $\eta_{v}$ from the zero net flux condition. We iterate this procedure until convergence.

Since mainly antineutrinos are affected by the changes in composition, we focus in Fig. 3 on the different contributions to the antineutrino absorption opacity $\left\langle\kappa_{\mathrm{abs}}\right\rangle$ and the effective opacity $\left\langle\kappa_{\text {eff }}\right\rangle$, at four different times post bounce. The dashed lines represent our reference calculation, which considers only antineutrino absorption on protons, nucleon-nucleon bremsstrahlung and neglects the presence of light nuclei, corresponding to the treatment in Ref. [7]. For comparison, the solid lines are based on the virial EOS composition and the antineutrino interactions as discussed above (similar results are obtained using the NSE EOS). We find in Fig. 3 that the antineutrino $\left\langle\kappa_{\mathrm{abs}}\right\rangle$ in the region around the neutrinosphere is dominated by the contribution from deuterons and tritons, for which charged-current and neutral-current reactions contribute approximately in equal amounts. Although tritons become more abundant (see Fig. 2), deuterons become more important for the opacities.

\section{NEUTRINO LUMINOSITIES, SPECTRA, AND NEUTRON EXCESS IN THE EJECTA}

In the following, we explore the influence of light nuclei on the properties of the emitted neutrinos. To this end, we consider four different cases denoted A through D in Table II, which use the different EOS discussed above. Our reference calculation is case $\mathrm{A}$, which is performed with the same EOS (consisting of neutrons, protons, and $\alpha$-particles in NSE) and the same neutrino reactions as in Ref. [7]. When we change 
TABLE II. Different cases explored in Sect. III.

\begin{tabular}{lcc}
\hline \hline Case & $Y_{e}$ determined from & EOS and composition \\
\hline A & beta equilibrium & NSE (n, $\left.\mathrm{p},{ }^{4} \mathrm{He}\right)$ \\
B & case A & NSE (nucleons and nuclei) \\
C & beta equilibrium & NSE (nucleons and nuclei) \\
D & beta equilibrium & virial (n, $\mathrm{p}, A \leqslant 4$ nuclei) \\
\hline \hline
\end{tabular}

to the improved EOS including light nuclei for given density, temperature, and $Y_{e}$, the different baryonic composition leads to a modified optical depth and therefore to a shift of mainly the position of the neutrinosphere of electron antineutrinos. On the other hand, for given density and temperature in the neutron star atmosphere, the improved EOS imply different nucleon chemical potentials and therefore yield a different value of $Y_{e}$ when we impose the constraint of beta equilibrium for the stellar matter (see Sec. II A). This again influences the composition, optical depth, and neutrinospheric positions. To quantify separately the impact due to the direct compostion change and the one associated with an adjustment to a new beta equilibrium, we define an intermediate case $B$ that uses the same density, temperature, and $Y_{e}$ profiles as case A but obtains the baryonic composition from the improved NSE EOS. Case $\mathrm{C}$ then uses the same EOS as case B but takes into account the adjustment to a new beta equilibrium. In case D, the baryonic composition as well as the beta equilibrium state are based on the virial EOS.

Table III shows the neutrinosphere radii and corresponding properties of electron neutrinos and antineutrinos for the four different cases. The average energies, defined as

$$
\left\langle\epsilon_{v}\right\rangle=\frac{F_{3}\left(\eta_{v}\right)}{F_{2}\left(\eta_{v}\right)} k T_{\nu},
$$

and luminosities computed for our reference case $\mathrm{A}$ are in good agreement with the results of Ref. [7]. In order to compare the average energies and luminosities of Table III with those commonly used in nucleosynthesis studies (which correspond to values measured at infinity), the gravitational redshift must be included. For our neutron star model, the redshift correction corresponds to a reduction by a factor of about 0.8 .

The differences in the various observables of Table III caused by the presence of light nuclei can be understood from a detailed comparison of the four cases. While case A considers the neutron star atmosphere to be in beta equilibrium with only neutrons, protons, and alpha particles, case $\mathrm{B}$ uses the same $Y_{e}$ profile as case A, but accounts for the presence of light nuclei. Their appearance happens at the expense of the number of free protons, whose mass fraction is drastically reduced. This leads to a lower antineutrino opacity than in case A, even when the additional antineutrino interactions with light nuclei are fully included. Consequently, the neutrinosphere of antineutrinos moves to a smaller radius where the temperature is larger so that antineutrinos are expected to be radiated with a higher mean energy.

However, as discussed above, the EOS with light nuclei also lead to a shift of the beta equilibrium conditions in the neutron star atmosphere. The balance of electron neutrino and antineutrino absorption and production reactions is locally established for a higher value of $Y_{e}$, thus compensating for

TABLE III. Neutrinosphere radii $R_{\bar{v}_{e}, v_{e}}$, neutrino spectral temperatures $T_{\bar{v}_{e}, v_{e}}$, and average energies $\left\langle\epsilon_{\bar{v}_{e}, v_{e}}\right\rangle$, as well as number luminosities $L_{n}$, spectral parameter $\eta_{v_{e}}$, and wind electron fractions $Y_{e}^{\mathrm{w}}$ at four different times post bounce.

\begin{tabular}{|c|c|c|c|c|c|c|c|c|c|}
\hline & $R_{\bar{v}_{e}}[\mathrm{~km}]$ & $T_{\bar{v}_{e}}[\mathrm{MeV}]$ & $\left\langle\epsilon_{\bar{v}_{e}}\right\rangle[\mathrm{MeV}]$ & $L_{n}\left[10^{56} \mathrm{~s}^{-1}\right]$ & $\eta_{v_{e}}$ & $R_{v_{e}}[\mathrm{~km}]$ & $T_{v_{e}}[\mathrm{MeV}]$ & $\left\langle\epsilon_{v_{e}}\right\rangle[\mathrm{MeV}]$ & $Y_{e}^{\mathrm{w}}$ \\
\hline \multicolumn{10}{|c|}{$t=2 \mathrm{~s}$} \\
\hline A & 10.01 & 8.14 & 25.64 & 6.05 & 0.72 & 10.55 & 6.34 & 20.71 & 0.514 \\
\hline B & 9.977 & 8.30 & 26.16 & 6.38 & 0.79 & 10.55 & 6.34 & 20.80 & 0.507 \\
\hline $\mathrm{C}$ & 10.00 & 8.17 & 25.73 & 6.10 & 0.73 & 10.55 & 6.35 & 20.75 & 0.513 \\
\hline D & 9.979 & 8.29 & 26.12 & 6.36 & 0.77 & 10.53 & 6.37 & 20.87 & 0.509 \\
\hline \multicolumn{10}{|c|}{$t=5 \mathrm{~s}$} \\
\hline A & 9.272 & 7.17 & 22.60 & 3.55 & 1.01 & 9.821 & 5.14 & 17.10 & 0.478 \\
\hline B & 9.260 & 7.24 & 22.83 & 3.65 & 1.04 & 9.819 & 5.15 & 17.16 & 0.475 \\
\hline $\mathrm{C}$ & 9.295 & 7.04 & 22.17 & 3.37 & 0.94 & 9.814 & 5.16 & 17.07 & 0.487 \\
\hline $\mathrm{D}$ & 9.272 & 7.17 & 22.60 & 3.55 & 1.00 & 9.813 & 5.16 & 17.15 & 0.480 \\
\hline \multicolumn{10}{|c|}{$t=7 \mathrm{~s}$} \\
\hline A & 9.107 & 6.88 & 21.69 & 3.03 & 1.15 & 9.683 & 4.73 & 15.90 & 0.462 \\
\hline B & 9.095 & 6.97 & 21.95 & 3.13 & 1.19 & 9.681 & 4.74 & 15.96 & 0.458 \\
\hline $\mathrm{C}$ & 9.139 & 6.68 & 21.04 & 2.78 & 1.04 & 9.676 & 4.75 & 15.82 & 0.475 \\
\hline D & 9.134 & 6.71 & 21.14 & 2.82 & 1.05 & 9.675 & 4.75 & 15.85 & 0.473 \\
\hline \multicolumn{10}{|c|}{$t=10 \mathrm{~s}$} \\
\hline A & 9.041 & 6.94 & 21.86 & 3.06 & 1.49 & 9.592 & 4.37 & 15.05 & 0.431 \\
\hline B & 9.039 & 7.02 & 22.12 & 3.17 & 1.53 & 9.590 & 4.37 & 15.12 & 0.427 \\
\hline $\mathrm{C}$ & 9.063 & 6.49 & 20.44 & 2.51 & 1.23 & 9.582 & 4.39 & 14.82 & 0.456 \\
\hline $\mathrm{D}$ & 9.065 & 6.45 & 20.32 & 2.47 & 1.20 & 9.581 & 4.39 & 14.80 & 0.458 \\
\hline
\end{tabular}




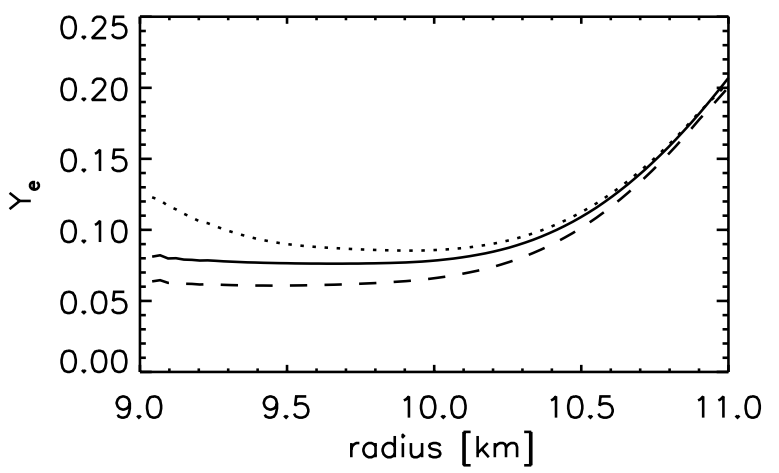

FIG. 4. Profile of the electron fraction $Y_{e}$ in the region around the neutrinosphere at $t=2 \mathrm{~s}$ after core bounce. The dashed line corresponds to the EOS of Ref. [7] (case A), the dotted line to the NSE EOS (case C), and the solid line to the virial EOS (case D). All profiles are obtained assuming beta equilibrium for the corresponding EOS.

the reduced abundance of free protons in the presence of light nuclei. This effect is shown in Fig. 4 for the $Y_{e}$ profile at $t=2 \mathrm{~s}$ from the different EOS. The shift of the beta equilibrium is taken into account in cases $\mathrm{C}$ and $\mathrm{D}$. As a consequence of the higher $Y_{e}$, the abundances of protons, deuterons, and tritons are larger than in case B (although free protons are clearly reduced relative to their abundance in case A, see Fig. 2). Compared to case B, the antineutrino opacity is therefore increased and the corresponding neutrinosphere is located at a larger radius, leading to a lower mean energy of the escaping antineutrinos.

In contrast to the properties of electron antineutrinos, the spectra of electron neutrinos are only slightly affected by the improved EOS and by the adjustment to a new beta equilibrium. There are two reasons for this: The mass fraction of free neutrons dominates the composition and only slightly differs for all cases, and second, weak reactions with neutrons are responsible for most of the opacity of electron neutrinos.

From this general discussion we conclude that the change of the baryonic composition due to light nuclei on the one hand and due to the neutrino-driven adjustment of matter to a new beta equilibrium on the other have effects in opposite directions for the position of the antineutrinosphere (and rather unimportant effects on the neutrinosphere). This makes it difficult to reliably predict the change of this position in cases $\mathrm{C}$ and $\mathrm{D}$ compared to the reference case A. In fact, as Table III shows, the outcome of these competing effects can go either way. At early times ( $t=2 \mathrm{~s}$ after bounce) the matter in the vicinity of the neutrinosphere has large temperatures and hence a rather large fraction of free protons is present (see Fig. 2). Under such conditions protons dominate the antineutrino opacity in the region around the antineutrinosphere (see Fig. 3). In cases $C$ and $D$ the proton mass fraction is noticeably reduced, but the additional opacity contributions due to light nuclei cannot compensate for the reduction of the proton opacity. As a result the antineutrinosphere moves to slightly smaller radii resulting in larger average energies for the emitted antineutrinos. As the protoneutron star and its atmosphere cool, the surface density profile steepens (see Fig. 1), and the neutrinospheres move to smaller radii within the same model. The matter in the region of the neutrinospheres becomes more neutron-rich and the proton abundance is lower. However, in cases C and D substantial amounts of deuterons and tritons are present in this region overcompensating the reduction in the proton mass fraction and making the total antineutrino absorption opacities higher in cases $\mathrm{C}$ and $\mathrm{D}$ compared to case A. Consequently, the antineutrinosphere moves to larger radii resulting in smaller antineutrino average energies.

We emphasize that at late times the antineutrinosphere is located at densities $\rho \gtrsim 10^{13} \mathrm{~g} / \mathrm{cm}^{3}$, where nuclear interactions and many-body contributions affect the composition and neutrino cross sections. For example, for $t=7$ and $10 \mathrm{~s}$, the contribution from densities $\rho>10^{13} \mathrm{~g} / \mathrm{cm}^{3}$ to the optical depth is $52 \%$ and $65 \%$, respectively. In the present work, as well as in state-of-the-art studies of protoneutron star winds [7], such potentially important effects have been neglected. They should be considered in future work.

In order to quantify the effects of the changing antineutrino energies on the nucleosynthesis conditions in the baryonic wind driven by neutrino energy deposition off the neutron star surface, we estimate the wind electron fraction $Y_{e}^{\mathrm{w}}$ from the expression [44]

$$
Y_{e}^{\mathrm{w}}=\frac{\lambda_{v_{e} n}}{\lambda_{v_{e} n}+\lambda_{\bar{v}_{e} p}} .
$$

Here $\lambda_{v_{e} n}$ and $\lambda_{\bar{v}_{e} p}$ are the neutrino absorption rate on neutrons and the antineutrino absorption rate on protons, respectively, which depend on the neutrino number luminosities $L_{n, v}$, the neutrino spectra, and the radial distance $r$ from the neutron star: $\lambda_{v}=L_{n, v}\langle\sigma\rangle /\left[4 \pi r^{2} f(r)\right]$, where $\langle\sigma\rangle$ is the relevant cross section suitably averaged over the neutrino spectrum $[4,44]$ and including weak magnetism corrections [29]. In making use of Eq. (7), we assume that the ejected matter is initially composed only of neutrons and protons, so we neglect the presence of alpha particles and the so-called alpha-effect [45]. Furthermore, we suppose that the matter is exposed long enough to neutrino and antineutrino captures to achieve an equilibrium between neutrino and antineutrino absorptions, and that this happens at such large distances (and low temperatures) that electron and positron captures can be ignored.

Using Eq. (7), we have calculated the wind electron fraction $Y_{e}^{\mathrm{w}}$ for the different cases. The corresponding results are given in Table III. Because of the increase of the average antineutrino energies, we find that matter is ejected in cases C and D at early times with slightly lower $Y_{e}^{\mathrm{w}}$ values than in case A. At later times ( $t \gtrsim 5 \mathrm{~s}$ after bounce) the mean antineutrino energies are smaller in cases $\mathrm{C}$ and $\mathrm{D}$ than in case $\mathrm{A}$, and therefore $Y_{e}^{\mathrm{w}}$ is slightly higher than in our reference case.

Once the ejected matter has reached larger radii and thus low enough temperatures, nuclei can form. During the early phases of nucleosynthesis mainly alpha particles but also light nuclei are present in the composition. Neutrino interactions with these light elements can constitute an additional source of energy deposition in the wind. As noted by Qian and Woosley [4] such an additional source of energy could increase the entropy and reduce the dynamical time scale of the wind, and consequently facilitate the production of heavy nuclei via the r-process [18]. Using one of the wind trajectories resulting from the hydrodynamical simulations of Ref. [7], we have computed the contribution to the energy deposition rate arising 
from light nuclei (deuterons, tritons, and alpha particles). Among the light nuclei, we found that the dominating contribution comes from neutrino interactions with alpha particles (where we have taken the cross sections from Gazit and Barnea [46]). However, our calculation showed that the additional energy deposition provided by light elements is too low, by more than an order of magnitude, to have an impact on the conditions for r-process nucleosynthesis.

\section{CONCLUSIONS}

We have shown that the thermodynamical conditions in the outer layers of a protoneutron star favor the presence of light nuclei, mainly deuterons and tritons, which are not accounted for by EOS currently used in core-collapse supernova simulations [13] and in studies of neutrino-driven supernova outflows [7]. Using the profiles of a hydrodynamical model for neutrino-driven supernova ejecta [7], we have estimated the effects of light nuclei on the emission of electron neutrinos and antineutrinos. For this purpose we have compared the virial and NSE EOS, which include light nuclei, to a reference case composed of neutrons, protons, and alpha particles.

The abundance of light nuclei can be studied in laboratory heavy-ion collisions. These experiments can reproduce the densities and temperatures near the neutrinosphere. In the future, because the neutrinosphere is neutron rich, the abundances of light nuclei should be measured for more neutron-rich systems. This can be done with radioactive beams.

The appearance of light nuclei has only a minor impact on the position of the electron neutrinosphere and consequently on the average energy of the radiated electron neutrinos. However, the situation is different for electron antineutrinos. At early times when the protoneutron star is relatively hot and protons have mass fractions around 0.1 in the neutrinospheric region, the appearance of light nuclei reduces the antineutrino opacity. Therefore, antineutrinos escape from hotter layers in the protoneutron star with slightly larger average energy. At later times the mass fraction of protons in the protoneutron star is greatly reduced, but light elements (in particular tritons) can have mass fractions that reach values even around 0.1 . This makes light nuclei the major source of opacity for antineutrinos. Comparing with the EOS used in Ref. [7], we see that antineutrinos are kept in thermal equilibrium with matter until larger radii in the protoneutron star, reducing the average energy of the emitted antineutrinos. For the latest time considered in the present study ( $t=10 \mathrm{~s}$ after bounce), the reduction could be as large as $1.5 \mathrm{MeV}$.

The changes in the antineutrino average energies can have consequences for the nucleosynthesis occurring in neutrinodriven winds. Such winds are a very interesting nucleosynthesis site. They allow for proton-rich ejecta during the first couple of seconds $[47,48]$, where the recently suggested $v p$-process $[49,50]$ may take place, as well as neutron-rich ejecta at later times, which might provide the conditions for r-process nucleosynthesis [51]. During the early proton-rich phase, the changes in the antineutrino energies are minor and consequently the electron-to-baryon ratio $Y_{e}^{\mathrm{w}}$ in the wind remains the same or is slightly reduced. For the neutron-rich phase and in particular for the latest times considered in the present study, we find that $Y_{e}^{\mathrm{w}}$ can increase by as much as 0.025. This is a substantial change, and if everything else remains the same, such a change makes the occurrence of strong r-processing less likely.

For more detailed studies, reliable estimates of the neutrino cross sections with light nuclei are desirable. The neutrinodeuteron cross sections [32] used here are probably sufficiently accurate at the neutrino energies considered in this work. For charged-current and neutral-current cross sections of antineutrino reactions with tritons, we have presented results based on a relatively simple RPA approach. The neutral-current cross sections were found to agree very well with the recent $a b$ initio results of Ref. [8]. Similar calculations for chargedcurrent cross sections would be useful.

Our estimates show that future simulations of neutrinodriven supernova outflows should take into account light elements in the baryonic composition of the stellar medium and the corresponding cross sections of neutrino interactions, especially those of electron antineutrinos. This is particularly important to fully quantify the consequences for the properties of the neutrino emission from forming neutron stars and for the neutrino-generated nucleosynthesis conditions in the baryonic mass that is lost from such stars. In addition to including light nuclei in the EOS, our results show that, for late times, an important future problem is understanding the properties of and neutrino interactions with nucleonic matter at densities above $10^{13} \mathrm{~g} / \mathrm{cm}^{3}$.

\section{ACKNOWLEDGMENTS}

A.A. acknowledges support by the Deutsche Forschungsgemeinschaft through contract SFB 634 "Nuclear structure, nuclear astrophysics and fundamental experiments at small momentum transfers at the S-DALINAC". The work of E.O. and A.S. is supported by the Natural Sciences and Engineering Research Council of Canada (NSERC). TRIUMF receives federal funding via a contribution agreement through the National Research Council of Canada. The work of H.-T.J. is supported by the Deutsche Forschungsgemeinschaft through the Transregional Collaborative Research Centers SFB/TR 27 "Neutrinos and Beyond" and SFB/TR 7 "Gravitational Wave Astronomy", and the Cluster of Excellence EXC 153 "Origin and Structure of the Universe" (http://www.universecluster.de). The work of C.J.H. is supported in part by DOE grant DE-FG02-87ER40365.
[1] H. Duan, G. M. Fuller, J. Carlson, and Y.-Z. Qian, Phys. Rev. Lett. 100, 021101 (2008).

[2] C. Lunardini, B. Mueller, and H. Janka (2007), arXiv:0712.3000 [astro-ph].
[3] R. C. Duncan, S. L. Shapiro, and I. Wasserman, Astrophys. J. 309, 141 (1986).

[4] Y.-Z. Qian and S. E. Woosley, Astrophys. J. 471, 331 (1996).

[5] J. M. Lattimer and F. D. Swesty, Nucl. Phys. A535, 331 (1991). 
[6] H. Shen, H. Toki, K. Oyamatsu, and K. Sumiyoshi, Nucl. Phys. A637, 435 (1998).

[7] A. Arcones, H.-T. Janka, and L. Scheck, Astron. Astrophys. 467, 1227 (2007).

[8] E. O'Connor, D. Gazit, C. J. Horowitz, A. Schwenk, and N. Barnea, Phys. Rev. C 75, 055803 (2007).

[9] K. Sumiyoshi and G. Röpke, Phys. Rev. C 77, 055804 (2008).

[10] G. Baym, H. A. Bethe, and C. J. Pethick, Nucl. Phys. A175, 225 (1971).

[11] M. Schmidt, G. Röpke, and H. Schulz, Ann. Phys. (NY) 202, 57 (1990).

[12] H. A. Bethe and J. R. Wilson, Astrophys. J. 295, 14 (1985).

[13] H.-T. Janka, K. Langanke, A. Marek, G. Martínez-Pinedo, and B. Müller, Phys. Rep. 442, 38 (2007).

[14] N. Ohnishi, K. Kotake, and S. Yamada, Astrophys. J. 667, 375 (2007).

[15] T. A. Thompson, A. Burrows, and B. S. Meyer, Astrophys. J. 562, 887 (2001).

[16] B. S. Meyer, G. J. Mathews, W. M. Howard, S. E. Woosley, and R. D. Hoffman, Astrophys. J. 399, 656 (1992).

[17] S. E. Woosley and R. D. Hoffman, Astrophys. J. 395, 202 (1992).

[18] R. D. Hoffman, S. E. Woosley, and Y.-Z. Qian, Astrophys. J. 482, 951 (1997).

[19] A. Burrows, L. Dessart, C. D. Ott, and E. Livne, Phys. Rep. 442, 23 (2007).

[20] S. Kowalski, J. B. Natowitz, S. Shlomo, R. Wada, K. Hagel, J. Wang, T. Materna, Z. Chen, Y. G. Ma, L. Qin et al., Phys. Rev. C 75, 014601 (2007).

[21] C. J. Horowitz and A. Schwenk, Nucl. Phys. A776, 55 (2006).

[22] C. J. Horowitz and A. Schwenk, Phys. Lett. B638, 153 (2006).

[23] E. O'Connor, A. Schwenk, and C. J. Horowitz (2008, in preparation).

[24] T. Rauscher, Astrophys. J. Suppl. 147, 403 (2003).

[25] W. R. Hix and F.-K. Thielemann, Astrophys. J. 460, 869 (1996).

[26] E. Bravo and D. García-Senz, Mon. Not. R. Astron. Soc. 307, 984 (1999).

[27] M. T. Keil, G. G. Raffelt, and H.-T. Janka, Astrophys. J. 590, 971 (2003).

[28] S. L. Shapiro and S. A. Teukolsky, Black Holes, White Dwarfs and Neutron Stars: The Physics of Compact Objects (WileyInterscience, New York, 1983).
[29] C. J. Horowitz, Phys. Rev. D 65, 043001 (2002).

[30] A. Burrows, S. Reddy, and T. A. Thompson, Nucl. Phys. A777, 356 (2006).

[31] S. Hannestad and G. Raffelt, Astrophys. J. 507, 339 (1998).

[32] S. Nakamura, T. Sato, S. Ando, T.-S. Park, F. Myhrer, V. Gudkov, and K. Kubodera, Nucl. Phys. A707, 561 (2002), see http://www. physics.sc.edu/ gudkov/NU-D-NSGK/; S. Nakamura, private communication.

[33] D. L. Tubbs and D. N. Schramm, Astrophys. J. 201, 467 (1975).

[34] E. Kolbe and K. Langanke, Phys. Rev. C 63, 025802 (2001).

[35] E. Kolbe, K. Langanke, G. Martínez-Pinedo, and P. Vogel, J. Phys. G: Nucl. Part. Phys. 29, 2569 (2003).

[36] E. Kolbe, K. Langanke, and P. Vogel, Nucl. Phys. A652, 91 (1999).

[37] J. D. Walecka, in Muon Physics, edited by V. W. Hughes and C. S. Wu (Academic Press, New York, 1975), Vol. II, Sect. V.4, pp. 113-218.

[38] T. W. Donnelly and R. P. Peccei, Phys. Rep. 50, 1 (1979).

[39] G. Martínez-Pinedo, A. Poves, E. Caurier, and A. P. Zuker, Phys. Rev. C 53, R2602 (1996).

[40] D. Mihalas, Stellar Atmospheres (W. H. Freeman and Co., San Francisco, 1978), 2nd ed., p. 62.

[41] H.-T. Janka, Astron. Astrophys. 244, 378 (1991).

[42] J. A. Pons, S. Reddy, M. Prakash, J. M. Lattimer, and J. A. Miralles, Astrophys. J. 513, 780 (1999).

[43] W. Keil and H.-T. Janka, Astron. Astrophys. 296, 145 (1995).

[44] Y.-Z. Qian, Prog. Part. Nucl. Phys. 50, 153 (2003).

[45] B. S. Meyer, G. C. McLaughlin, and G. M. Fuller, Phys. Rev. C 58, 3696 (1998).

[46] D. Gazit and N. Barnea, Phys. Rev. Lett. 98, 192501 (2007).

[47] J. Pruet, S. E. Woosley, R. Buras, H.-T. Janka, and R. D. Hoffman, Astrophys. J. 623, 325 (2005).

[48] C. Fröhlich, P. Hauser, M. Liebendörfer, G. Martínez-Pinedo, F.-K. Thielemann, E. Bravo, N. T. Zinner, W. R. Hix, K. Langanke, A. Mezzacappa et al., Astrophys. J. 637, 415 (2006).

[49] C. Fröhlich, G. Martínez-Pinedo, M. Liebendörfer, F.-K. Thielemann, E. Bravo, W. R. Hix, K. Langanke, and N. T. Zinner, Phys. Rev. Lett. 96, 142502 (2006).

[50] J. Pruet, R. D. Hoffman, S. E. Woosley, H.-T. Janka, and R. Buras, Astrophys. J. 644, 1028 (2006).

[51] M. Arnould, S. Goriely, and K. Takahashi, Phys. Rep. 450, 97 (2007). 